\title{
Adipocyte differentiation-related protein promotes lipid accumulation in goat mammary epithelial cells
}

\author{
H. B. Shi, ${ }^{+} \dagger^{1}$ K. Yu, ${ }^{* 1}$ J. Luo, ${ }^{* 2}$ J. Li, ${ }^{*}$ H. B. Tian, ${ }^{*}$ J. J. Zhu, ${ }^{*}$ Y. T. Sun, ${ }^{*}$ D. W. Yao, ${ }^{*}$ H. F. Xu, ${ }^{*}$ H. P. Shi, ${ }^{*}$ \\ and J. J. Loor ${ }^{2}$ \\ *Shaanxi Key Laboratory of Molecular Biology for Agriculture, College of Animal Science and Technology, Northwest A\&F University, Yangling, \\ Shaanxi, P.R. China 712100 \\ †College of Life Science, Zhejiang Sci-Tech University, Hangzhou, Zhejiang, P. R. China 310018 \\ ¥Mammalian NutriPhysioGenomics, Department of Animal Sciences and Division of Nutritional Sciences, University of Illinois, Urbana 61801
}

\begin{abstract}
Milk fat originates from the secretion of cytosolic lipid droplets (CLD) synthesized within mammary epithelial cells. Adipocyte differentiation-related protein (ADRP; gene symbol PLIN2) is a CLD-binding protein that is crucial for synthesis of mature CLD. Our hypothesis was that ADRP regulates CLD production and metabolism in goat mammary epithelial cells (GMEC) and thus plays a role in determining milk fat content. To understand the role of ADRP in ruminant milk fat metabolism, ADRP (PLIN2) was overexpressed or knocked down in GMEC using an adenovirus system. Immunocytochemical staining revealed that ADRP localized to the surface of CLD. Supplementation with oleic acid (OA) enhanced its colocalization with CLD surface and enhanced lipid accumulation. Overexpression of ADRP increased lipid accumulation and the concentration of triacylglycerol in GMEC. In contrast, morphological examination revealed that knockdown of ADRP decreased lipid accumulation even when OA was supplemented. This response was confirmed by the reduction in mass of cellular TG when ADRP was knocked down. The fact that knockdown of ADRP did not completely eliminate lipid accumulation at a morphological level in GMEC without OA suggests that some other compensatory factors may also aid in the process of CLD formation. The ADRP reversed the decrease of CLD accumulation induced by adipose triglyceride lipase. This is highly suggestive of ADRP promoting triacylglycerol stability within CLD by preventing access to adipose triglyceride lipase. Collectively, these data provide direct in vitro evidence that ADRP plays a key role in CLD formation and stability in GMEC.
\end{abstract}

\footnotetext{
Received February 10, 2015.

Accepted May 21, 2015.

${ }^{1}$ These authors contributed equally to this paper.

${ }^{2}$ Corresponding authors: luojun@nwsuaf.edu.cn and jloor@illinois.
} edu
Key words: milk fat, lipid droplet, triacylglycerol, hydrolysis

\section{INTRODUCTION}

The lactating mammary gland is one of the most lipogenic organs of the female body in many species. The majority of the lipid produced during lactation is secreted into milk by a process of membrane envelopment of cytoplasmic lipid droplets (CLD; Hansen et al., 1984; Chong et al., 2011). The CLD are composed of a sterol esters and triacylglycerol (TG) core surrounded by a phospholipid monolayer and coated with specific proteins (Krahmer et al., 2009; Wilfling et al., 2014). Several proteins have been identified on the lipid droplet (LD; Reinhardt and Lippolis, 2006; Maller et al., 2010; Lu et al., 2014), including perilipin 1 ( PLIN1), adipocyte differentiation-related protein ( ADRP; also known as PLIN2), and tail-interacting 47-kDa protein (TIP47; also known as PLIN3), which are known as the PAT domain proteins (Brasaemle et al., 2004; Thiele and Spandl, 2008; Russell et al., 2011). Proteomic studies confirmed that ADRP is a major protein component of LD in the mammary gland ( $\mathrm{Wu}$ et al., 2000; Lu et al., 2014), suggesting that it plays a role in the formation of $\mathrm{LD}$ during lactation.

Adipocyte differentiation-related protein is a $50-\mathrm{kDa}$ protein initially described in adipocytes (Jiang and Serrero, 1992) and considered a marker of lipid accumulation. Ubiquitously expressed, ADRP participates in CLD formation in hepatocytes (Motomura et al., 2006), adipocytes (Brasaemle et al., 1997), mammary epithelial cells (Russell et al., 2011), fibroblasts (Imamura et al., 2002), and macrophages (Larigauderie et al., 2006). In adipocytes, ADRP is present at the surface of CLD and is the major protein during early stages of adipocyte differentiation. During adipocyte differentiation, even though ADRP mRNA remains elevated, its protein level is reduced dramatically after the onset of perilipin expression (Jiang and Serrero, 1992; Bickel et al., 2009). 
Overexpression of the gene encoding ADRP in Swiss3T3 cells increased the number and size of CLD without induction of other adipocyte-specific or lipogenic genes (Imamura et al., 2002), providing direct evidence that it can modulate CLD levels. However, the fact that ADRP-deficient mice displayed normal adipocyte and adipose tissue differentiation and function, but had reduced liver TG content with attenuation of hepatic steatosis induced by high-fat feeding (Chang et al., 2006), suggests that ADRP may not be a central factor modulating CLD in adipocytes.

Adipocyte differentiation-related protein plays roles in milk fat formation and secretion in mammary cells. At the mRNA and protein levels, ADRP in the lactating mammary gland increases over 30-fold compared with nonlactating mammary gland in mice (Russell et al., 2007), cows (Bionaz and Loor, 2008), and goats (Shi et al., 2015). Furthermore, the pattern of ADRP mRNA expression in the differentiating mammary gland correlates closely with the appearance, growth and accumulation of CLD in murine milk-secreting cells (Russell et al., 2007). Adipocyte differentiation-related protein deficiency in the mouse mammary gland did not prevent CLD formation; however, it disrupted the increase in CLD size (Russell et al., 2011).

Previous evidence implicates ADRP as a physiological regulator of milk lipid production. Beyond the role in lipid storage, ADRP is also hypothesized to participate in CLD secretion through formation of a tripartite complex with the transmembrane protein butyrophilin (BTN) and the ubiquitously-expressed cytoplasmic enzyme xanthine oxidoreductase (XOR) in mammary cells (McManaman et al., 2007; Chong et al., 2011). However, even though some evidence supports an important role of ADRP in milk fat formation, the mechanism of ADRP regulation of CLD metabolism in lactating mammary gland tissue is still unclear.

To date, most of the research about ADRP in lactating mammary gland was performed in rodents. Compared with rodents, less is known about how ADRP affects lipid accumulation in ruminants, despite the fact that ruminant animals produce the majority of milk and dairy products consumed by humans. To investigate the role of $A D R P$ in lipid metabolism in mammary cells, the $A D R P$ gene was knockdown or overexpressed in goat mammary epithelial cells (GMEC).

\section{MATERIALS AND METHODS}

\section{Adenovirus Generation}

Recombinant adenovirus with shRNA for the $A D R P$ gene knockdown (Ad-sh511) was generated as reported previously (Kang et al., 2015). Recombinant adeno- virus for overexpression of $A D R P$ (Ad-ADRP) and adipose triglyceride lipase ( $A T G L$; Ad-ATGL) were packed and amplified as described previously (Shi et al., 2013a, 2014; Li et al., 2015). Briefly, the cDNA of dairy goat ADRP (NCBI: HQ846827.1) and ATGL (NCBI: GQ918145) were subcloned into the pAdTrack-CMV plasmid vector (Invitrogen, Waltham, MA) between the XhoI and KpnI (New England Biolabs, Ipswich, MA) restriction sites to generate pAdTrack-CMV-ADRP and pAdTrack-CMV-ATGL vectors. These 2 vectors were inserted into an adenoviral vector (pAdEasy-1, Invitrogen) to generate adenoviral plasmids in BJ5183 cells. The adenoviral plasmids linearized by Pac I (New England Biolabs) were transfected into 293A cells to generate the adenovirus Ad-ADRP and Ad-ATGL. The adenovirus with green fluorescent protein (Ad-GFP) and small hairpin RNA (shRNA) negative control adenovirus (Ad-NC; noneffective control), gifts from Zhijie Chang (Tsinghua University, Beijing, China), were used as negative controls. In our study, all the virus vectors express green fluorescent protein.

\section{Cell Culture and Treatment}

The GMEC were isolated from peak lactation Xinong Saanen goats as described previously (Wang et al., 2010). The cells used in the experiments were isolated and pooled from at least 3 different individual goats. Details of cell culture were described recently (Lin et al., 2013; Shi et al., 2013b). Briefly, cells were incubated at $37^{\circ} \mathrm{C}$ in $5 \% \mathrm{CO}_{2}$ and air. Culture medium was changed every $24 \mathrm{~h}$. Medium was composed of DMEM/F12 (Hyclone, Beijing, China) containing insulin $(5 \mathrm{mg} / \mathrm{L}$, Sigma-Aldrich, St. Louis, MO), hydrocortisone $(5 \mathrm{mg} / \mathrm{L}$, Sigma-Aldrich), penicillin and streptomycin $(10,000$ U/L, Harbin Pharmaceutical Group, Harbin, China), epidermal growth factor (1 mg/L, Sigma-Aldrich), and fetal bovine serum (10\%, Gibco, Grand Island, NY). The GMEC were transduced with adenovirus supernatant (Ad-ADRP, Ad-GFP, Ad-sh511, Ad-NC, or Ad-ATGL) until 70 to $80 \%$ confluence. Transfected GMEC were cultured with oleic acid (OA; O1008, Sigma-Aldrich) or ethyl alcohol (Sigma-Aldrich) after $24 \mathrm{~h}$ of the initial culture and then harvested at $48 \mathrm{~h}$ (24 h later) for RNA or protein extraction, quantification of total cellular triacylglycerol (TG) or oil red O staining. Oleic acid was diluted in ethyl alcohol and used at a $400 \mu M$ working concentration.

\section{RNA Extraction and Real-Time PCR}

Total cellular RNA was extracted from cells using RNA prep pure cell kit (Tiangen Biotech, Beijing, China) and prepared for reverse transcription using 
PrimeScript RT reagent kit (Takara, Shiga, Japan). Quantitative real-time PCR (qPCR) was performed with SYBR Premix Ex Taq II (Takara) on a CFX96 Real-time System (Bio-Rad, Hercules, CA). Relative mRNA expression levels of the target genes were normalized to glyceraldehyde-3-phosphate dehydrogenase $(G A P D H)$. The primers of qPCR for $A D R P$ and $G A P D H$ were described previously (Shi et al., 2013b).

\section{Western Blotting}

Western blotting was performed as previously described (Kang et al., 2015). Briefly, GMEC incubated with Ad-GFP or Ad-ADRP were collected at $48 \mathrm{~h}$. These samples were lysed with radioimmunoprecipitation assay (RIPA) buffer with a protease inhibitor mixture (Solarbio, Beijing, China). Protein concentrations were determined using a bicinchoninic acid (BCA) protein assay (Pierce, Rockford, IL) according to the manufacturer's instructions. The blots were incubated with a polyclonal antibody against ADRP (1:200; sc32888, Santa Cruz Biotechnology, Dallas, TX), and a monoclonal antibody against GAPDH $(1: 1,000$; Cwbiotech, Beijing, China). Secondary antibody used a goat anti-rabbit IgG with horseradish peroxidase or phycoerythrin (1:1,000; Transgen, Beijing, China). Signals were detected using a chemiluminescent ECL system (Pierce) and visualized by autoradiography with a cold CCD camera (Bio-Rad).

\section{Oil Red O Staining}

The GMEC cultured in 6-well plates (Nunc, Roskilde, Denmark) were incubated with adenovirus supernatant for $48 \mathrm{~h}$. Oleic acid was then added for an additional 24 $\mathrm{h}$ before harvesting. Oil Red O staining was performed on GMEC according to a method described previously (Mehlem et al., 2013) with modifications. Briefly, the samples were washed twice with PBS and fixed with $4 \%$ formaldehyde for $30 \mathrm{~min}$ at room temperature. After 2 washes in PBS, cells were stained with $\sim 1 \mathrm{~mL}$ of Oil Red O working solution $(3.5 \mathrm{~g} / \mathrm{L}$, diluted in isopropyl alcohol) for $15 \mathrm{~min}$ and then examined microscopically. The images were monitored using a Leica fluorescence microscope (DMI4000B, Leica, Wetzlar, Germany).

\section{Quantification of Total Cellular TG}

Total cellular TG was extracted according to the protocol of the GPO-Trinder triglyceride assay kit (Applygen Technologies, Beijing, China). Briefly, cells were collected and suspended in lysis buffer, following by sonication for $20 \mathrm{~s}$. After centrifuging the cell suspension at $10,000 \times g$ for $10 \mathrm{~min}$ at $4^{\circ} \mathrm{C}$, the supernatant was removed for TG and protein analysis. The lysis buffer was used as blank control to correct the values of samples and standards. The concentration of TG was determined according to the manufacturer's instructions on a microtiter plate reader (BioTek, Winooski, VT). The concentrations were calculated using the equation obtained from linear regression of the standard curve. The quantification of total cellular TG was normalized to the cellular protein concentration. Protein concentration of each well was determined using a BCA protein assay (Pierce) according to the manufacturer's instructions.

\section{Immunocytochemical Staining}

For the fluorescence experiments, GMEC were cultured on 6-well coverslip-bottomed plates (Nunc) and treated as described above. After harvesting, cells were fixed in $4 \%$ (vol/vol) paraformaldehyde (SigmaAldrich) for 15 min at room temperature. After a brief rinse in PBS, cells were treated with Triton-X100 of $0.5 \% \mathrm{w} / \mathrm{v}$ for $25 \mathrm{~min}$ at room temperature and then blocked before staining with $1 \%$ skim milk powder in the presence of $0.1 \% \mathrm{wt} / \mathrm{vol}$ of Tween-20 (all from Sigma-Aldrich) for $2 \mathrm{~h}$ at room temperature. Primary antibody incubation was performed overnight at $4^{\circ} \mathrm{C}$. Primary antibody for ADRP (rabbit polyclonal IgG, Santa Cruz Biotechnology) was diluted 1:200 in PBS with $1 \%$ dried bovine milk. After 3 washes with PBS with $0.1 \% \mathrm{wt} / \mathrm{vol}$ Tween-20, cells were incubated for $1 \mathrm{~h}$ with appropriate secondary antibodies (1:1,000; Cwbiotech), phycoerythrin-conjugated goat anti-rabbit (1:1,000 dilution), or fluorescein isothiocyanate (FITC)conjugated goat anti rabbit (1:1,000 dilution).

To visualize neutral lipids, $1 \mathrm{mg} / \mathrm{mL}$ of BODIPY 493/503 (Invitrogen) was added to cells during staining with the secondary antibody. To visualize the nucleus, after washing with PBS solution, the cells were incubated in 4',6-diamidino-2-phenylindole (DAPI; working solution, $1 \mu \mathrm{g} / \mathrm{mL}$, Invitrogen) for $15 \mathrm{~min}$ at $37^{\circ} \mathrm{C}$. The stained cells were visualized with a confocal laser microscope (A1R/A1, Nikon, Tokyo, Japan) using a $63 \times$ oil objective lens.

\section{Data Analysis}

Data for all measurements are from triplicate incubations, and all the experimental data are reported as means \pm standard error of the mean. Data of qPCR were analyzed relative to the control using the $2^{-\Delta \Delta \mathrm{Ct}}$ method, as described previously (Oppi-Williams et al., 2013), and differences among treatments determined via $t$-test (Shi et al., 2014). The data for total TG mass was normalized to the protein concentration before 
statistical analysis. One-way ANOVA (SAS Institute Inc., Cary, NC) was used for statistical comparisons and for determining the difference of total TG mass among treatments. The statistical model included the fixed effect of treatment. Differences were considered significant at $P<0.05$.

\section{RESULTS}

\section{Expression of ADRP Protein in GMEC}

The immunofluorescent staining was performed to detect the expression of ADRP in GMEC. As shown in Figure 1A, GMEC transfected with Ad-GFP expressed endogenous ADRP protein (stained red). Compared with the control group (Ad-GFP), Ad-ADRP significantly increased the expression of ADRP, which was confirmed by the qPCR and Western blotting (Figure 1B). Similar to our previous data from Western blotting (Kang et al., 2015; Supplementary Figure S1, http:// dx.doi.org/10.3168/jds.2015-9452), Ad-sh511 decreased the expression of ADRP compared with the control AdNC (Figure 1A). Based on these results, Ad-sh511 and Ad-ADRP were used to block and overexpress expression of ADRP in GMEC.

\section{ADRP Protein Co-Localizes with CLD in GMEC}

In this experiment, CLD were stained with BODIPY 493/503 and ADRP was stained with a secondary antibody with FITC. As shown in Figure 2A, most CLD accumulated around the nucleus (stained by DAPI). The ADRP protein colocalized with the CLD. In response to OA supplementation, the formation of CLD was stimulated markedly and filled the cells. The Oil Red O staining (Figure 2B) and quantification of total cellular TG $(P<0.001)$ further confirmed that OA promoted the increase of lipid accumulation and TG mass (Figure 2C).

\section{ADRP Enhances the Synthesis of CLD in GMEC}

After overexpression of ADRP, Oil Red O staining revealed that the neutral lipid accumulation was enhanced compared with the control group (Ad-GFP; Figure 3A). The OA markedly increased the cellular lipid accumulation in the presence or absence of AdADRP. Overexpression of ADRP in GMEC increased both the number and size of CLD, whereas both incubation with OA and Ad-ADRP had the strongest effect on the increase in number and size of CLD (Figure 3A). Consistent with the morphological changes, TG analysis confirmed that cells incubated with either ADRP or OA had higher TG concentrations compared with the control (Ad-GFP group; Figure 3C). In addition, overexpression of $A D R P$ further enhanced the concentration of TG induced by OA.

Compared with the control (Ad-NC), a significant morphological decrease in lipid accumulation was observed after knockdown of ADRP in cultures without OA supplementation (Figure 3A). Knockdown of $A D R P$ decreased the lipid accumulation even when OA was supplemented (Figure 3A). Furthermore, the quantification of total cellular TG revealed that cells incubated with Ad-sh511 had lower TG concentration compared with the control (Ad-NC; $P=0.010$ ). Despite the fact that OA enhanced the basal cellular lipid accumulation, knockdown of $A D R P$ still decreased the concentration of TG $(P=0.002$; Figure 3D).

Immunocytochemical staining was performed to evaluate the effect of $A D R P$ on lipid accumulation in GMEC. We observed that overexpression of ADRP increased the accumulation of lipid (Figure 3B). However, despite of the treatment with OA, the CLD decreased to the lowest concentration after knockdown of $A D R P$ (Figure 3B).

\section{ADRP Reduces Lipid Hydrolysis Induced by ATGL}

To explore the role of $A D R P$ on CLD stability and homeostasis, an Ad-ATGL adenovirus suspension was incubated with GMEC. The Oil Red O staining revealed that the OA treatment always enhanced the lipid accumulation and increased the total TG concentration of GMEC (Figure 4A, B, and C). Overexpression of $A T G L$ decreased the ability of the lipid accumulation and total TG level in GMEC (Figure 4A and B). However, ADRP could overcome the effect of $A T G L$, and increased the lipid accumulation (Figure $4 \mathrm{~A}$ ) and TG concentration $(P=0.039$; Figure $4 \mathrm{C})$. In the presence of OA, ADRP still had the ability to promote TG and lipid accumulation which was inhibited by $A T G L(P=$ $0.019)$. Such response was even more impressive given the high basal concentration of TG. In contrast, knockdown of ADRP further decreased the lipid accumulation and TG concentration in GMEC in the absence $(P$ $=0.029)$ or presence of OA $(P=0.04)$.

\section{DISCUSSION}

During lactation, the mammary gland synthesizes and packages TG into CLD that are subsequently secreted into milk (McManaman, 2012). The ADRP protein, one member of the PAT family, was identified as an important element for stabilization of CLD in adipocytes and nonadipocyte cells (Sztalryd et al., 2006; Thiele and Spandl, 2008). Despite the fact that lactating mammary tissue is the most lipogenic organ 
A

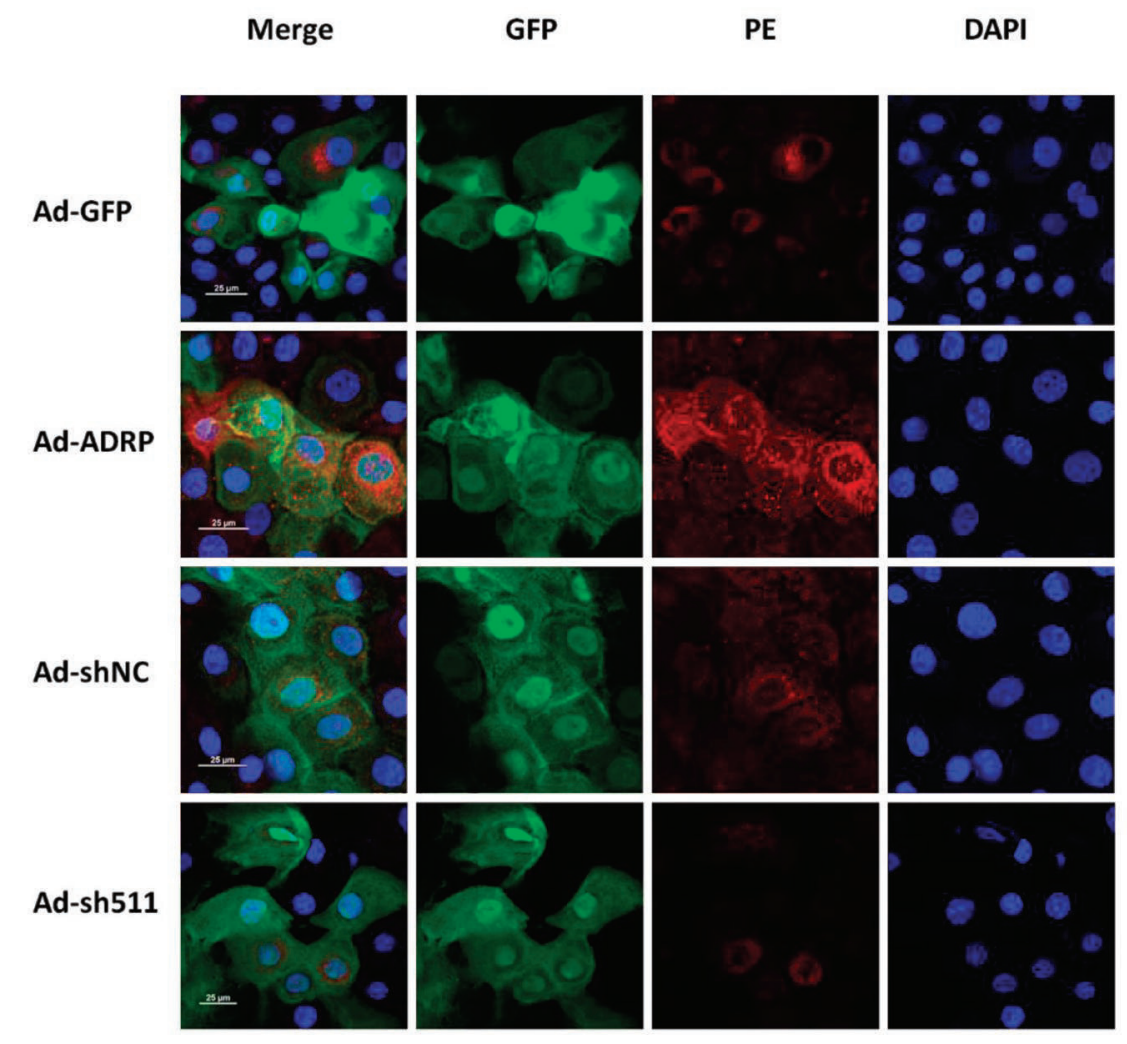

\section{B}
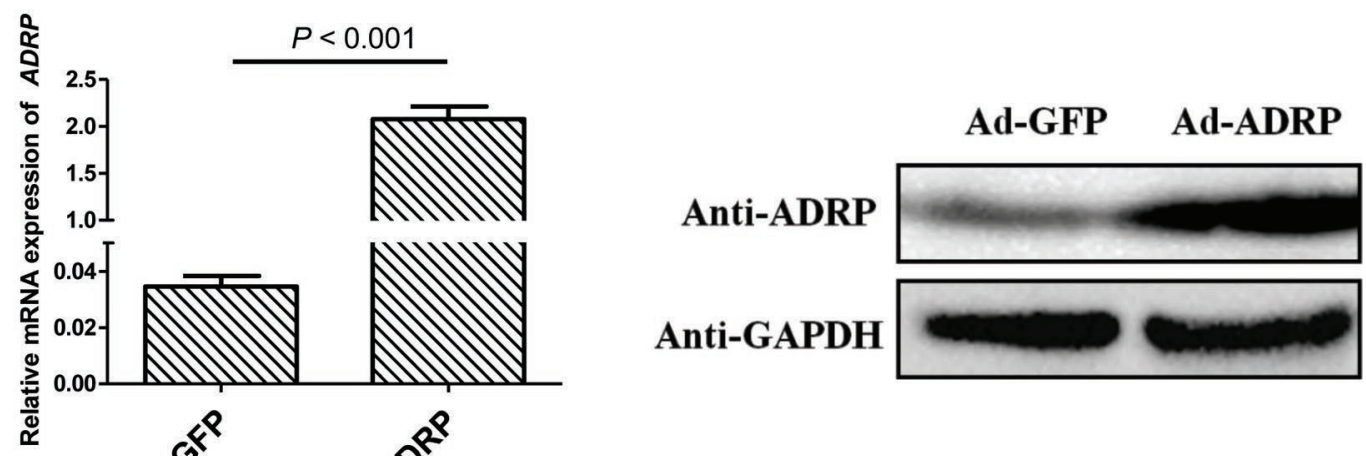

Figure 1. Adipocyte differentiation-related protein (ADRP) gene and protein expression in goat mammary epithelial cells (GMEC). Suspensions of adenovirus with shRNA (Ad-sh511), ADRP (Ad-ADRP), green fluorescent protein (Ad-GFP), or negative control (Ad-NC) were incubated with GMEC for $48 \mathrm{~h}$. The cells were then collected for immunofluorescent staining, quantitative real-time PCR (qPCR) or Western blot. The cells were collected for immunofluorescent staining, qPCR, or Western blot. (A) ADRP protein levels were detected by immunofluorescent staining with anti-ADRP antibody/phycoerythrin (PE)-conjugated anti-mouse (red). The nuclear was stained using 4',6-diamidino-2-phenylindole (DAPI; blue). The GFP protein (green) is used as control, which is expressed by the adenovirus. Fluorescent images were captured at the same spot in each column (bars $=25 \mu \mathrm{m}$ ). (B) Western blot and qPCR were used to detect the overexpression level of $A D R P$ in GMEC incubated with Ad-ADRP. Values are means \pm SEM for measurements of 3 wells. Difference was considered significant when $P<0.05$. Color version available online. 
A

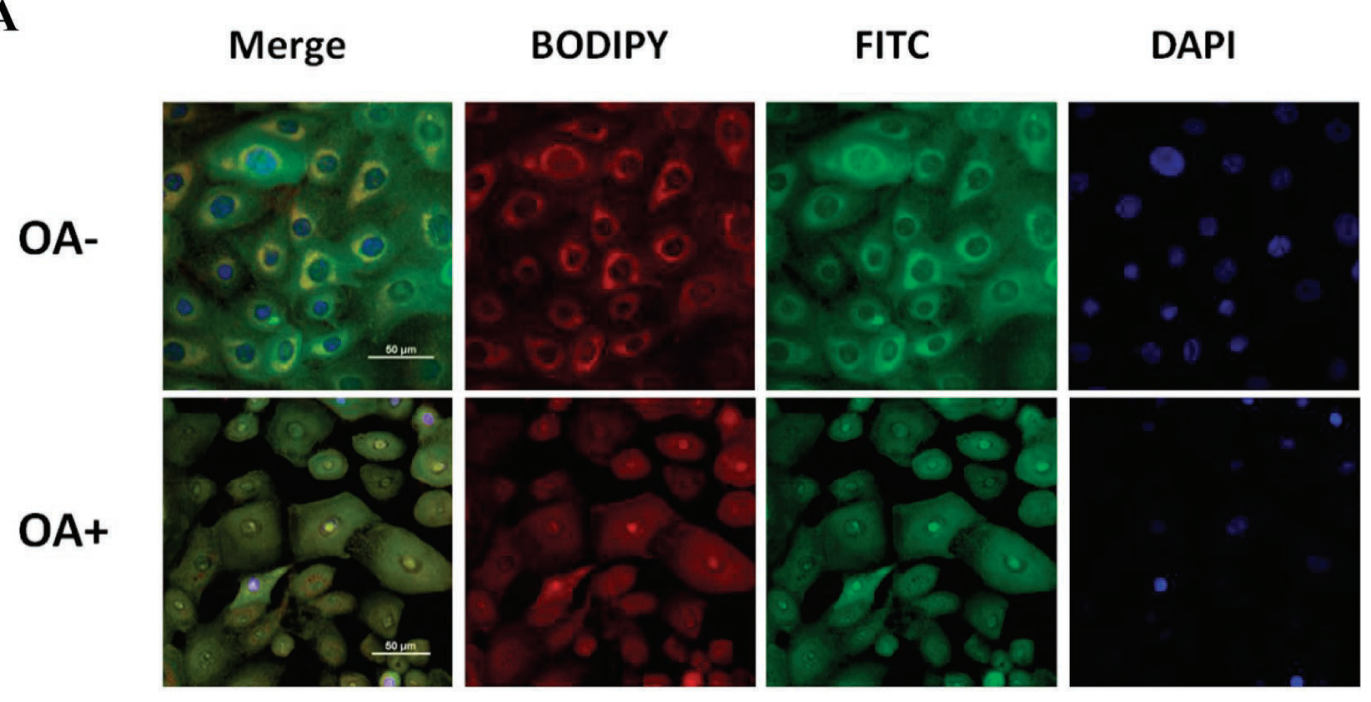

B

OA-

$\mathrm{OA}+$
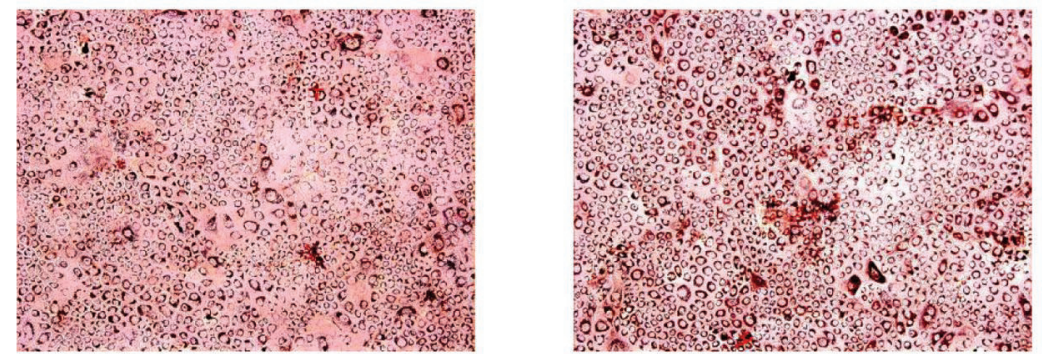

C

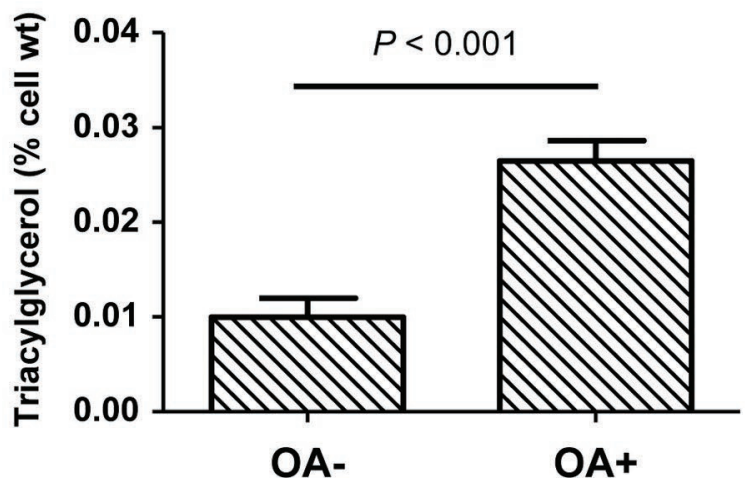

Figure 2. Oleic acid enhances the localization of adipocyte differentiation-related protein (ADRP) on the lipid droplet and increases the concentration of triacylglycerol in goat mammary epithelial cells (GMEC). The GMEC were incubated with $400 \mu M$ oleic acid (OA) for 24 $\mathrm{h}$, and then were collected for immunofluorescent staining and quantification of total cellular triacylglycerol. (A) Immunofluorescent staining was performed to detect the co-localization of ADRP protein and lipid droplet in GMEC incubated with OA or not. The protein was detected by with anti-ADRP antibody/fluorescein isothiocyanate (FITC)-conjugated anti-mouse (green). The nuclear was stained using 4',6-diamidino2-phenylindole (DAPI; blue). Lipid droplets were stained with BODIPY 493/503 (red; Invitrogen, Waltham, MA). Fluorescent images were captured at the same spot in each column (bars $=25 \mu \mathrm{m}$ ). (B) Oil Red O staining detection for lipid accumulation after incubation with OA $(\mathrm{OA}+)$ compared with control group $(\mathrm{OA}-)$. Images were captured at the same spot in each column (50× magnification). (C) Quantification of total triacylglycerol (TG) mass in OA treated GMEC. The concentration of TG was normalized to total protein. Values are means \pm SEM for measurements of 3 wells. Difference in relative total TG concentration was considered significant when $P<0.05$. Color version available online. 


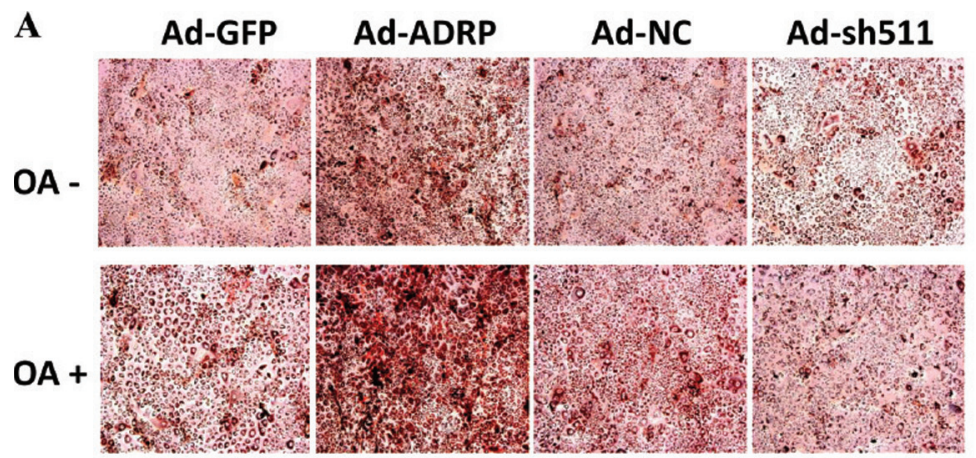

B

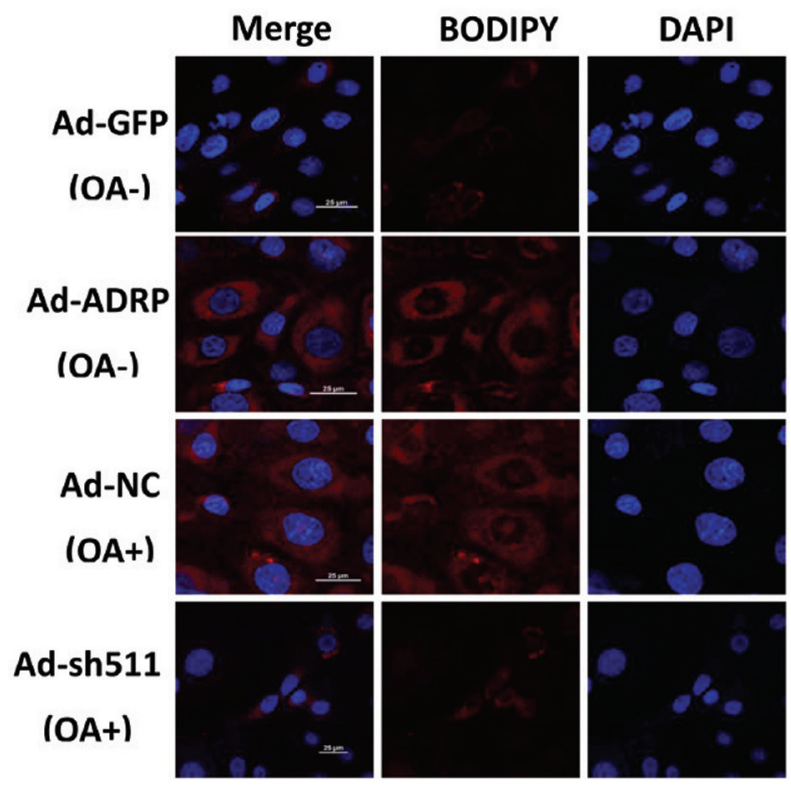

\section{C}

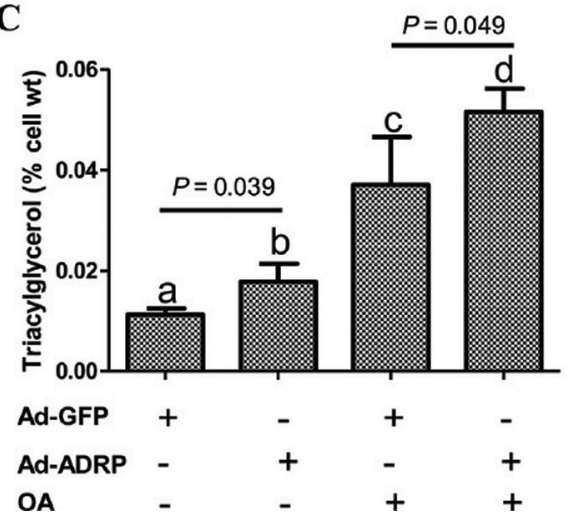

D

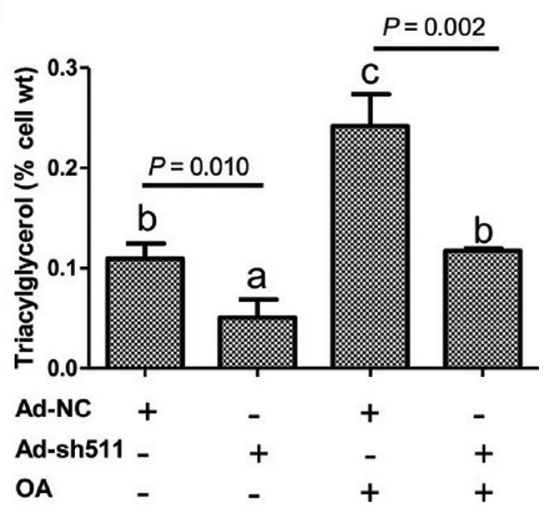

Figure 3. Adipocyte differentiation-related protein (ADRP) gene overexpression enhances the synthesis of lipid droplets in goat mammary epithelial cells (GMEC). The GMEC were incubated with adenovirus suspension [with shRNA (Ad-sh511), ADRP (Ad-ADRP), green fluorescent protein (Ad-GFP), or negative control (Ad-NC)] for $48 \mathrm{~h}$ with or without $400 \mu M$ oleic acid (OA) and then the cells was collected for fluorescence staining, Oil Red O staining and quantification of total triacylglycerol mass. (A) Fluorescence detection of alteration of lipid accumulation via knockdown or overexpression of ADRP in GMEC. The cells for fluorescence detection of lipid accumulation under treatment with Ad-GFP and Ad-ADRP were incubated without OA, and cells treated with Ad-NC and Ad-sh511 were incubated with OA. The nuclear was stained using 4',6-diamidino-2-phenylindole (DAPI; blue). Lipid droplets were stained with BODIPY 493/503 (red: Invitrogen, Waltham, MA). Fluorescent images were captured at the same spot in each column (bars $=25 \mu \mathrm{m}$ ). (B) Oil Red O staining detection for lipid accumulation after knockdown (Ad-sh511) or overexpression of ADRP (Ad-ADRP) compared with control groups (Ad-NC or Ad-GFP). Images were captured at the same spot in each column (50× magnification). (C) Relative total triacylglycerol (TG) mass in GMEC after overexpression of $A D R P$. (D) Relative total TG mass in GMEC after knockdown of ADRP. Values are means \pm SEM for measurements of 3 wells. The different letters $(\mathrm{a}-\mathrm{d})$ denote significant $(P<0.05)$ differences in relative total TG concentration. Difference in fold change was considered significant when $P<0.05$. Color version available online. 
A

Ad-ATGL+ Ad-ATGL+
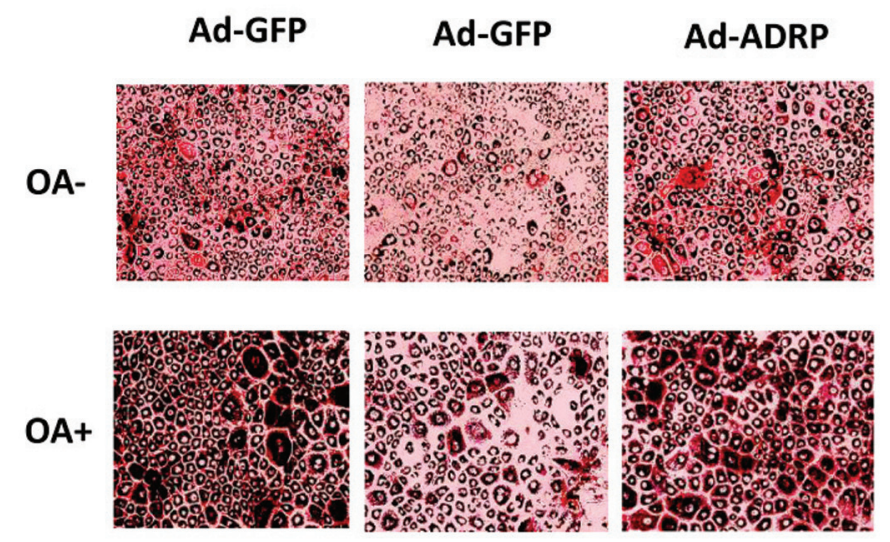

B

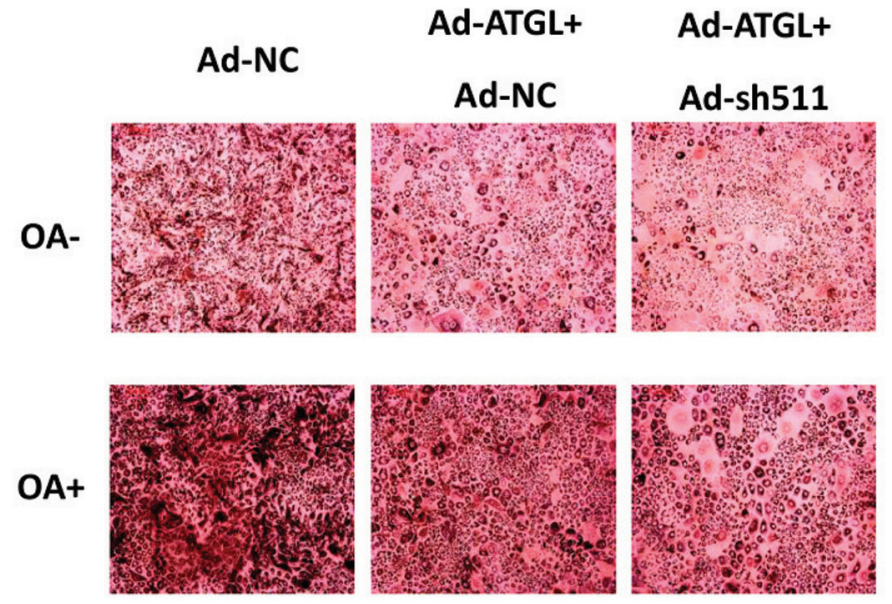

C

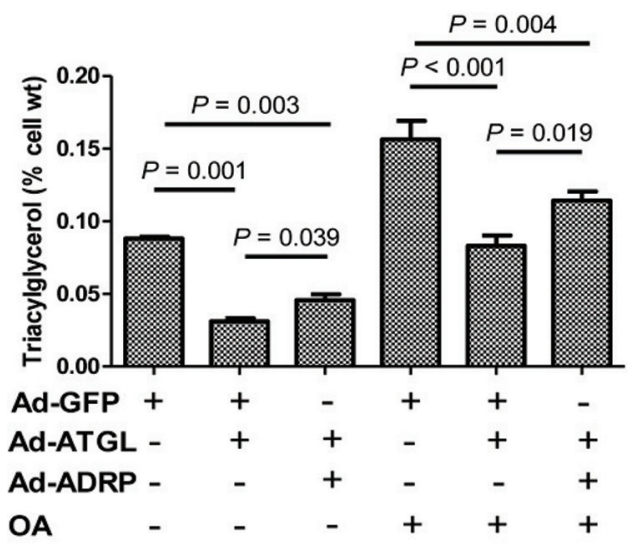

D

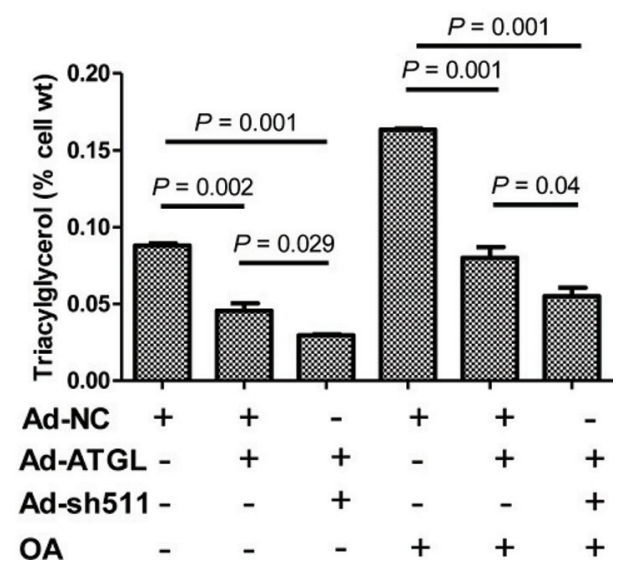

Figure 4. Adipocyte differentiation-related protein (ADRP) gene overexpression reduces lipid hydrolysis induced by adipose triglyceride lipase (ATGL) gene overexpression. Goat mammary epithelial cells (GMEC) were incubated with 1 or 2 adenovirus suspension [with ADRP (Ad-ADRP), green fluorescent protein (Ad-GFP), adipose triglyceride lipase (Ad-ATGL), negative control (Ad-NC), and shRNA (Ad-sh511)] for $48 \mathrm{~h}$ without or with oleic acid (OA) at $400 \mu \mathrm{M}$ and then the cells was collected for Oil Red O staining and quantification of total triacylglycerol (TG) mass. (A) Oil Red O staining detection for lipid accumulation after treatment with Ad-sh511, Ad-ADRP, or Ad-ATGL compared with control groups (Ad-NC or Ad-GFP). Images were captured at the same spot in each column (50× magnification). (B) Relative total TG mass in GMEC after overexpression of $A D R P$ and $A T G L$ along with or without OA. (C) Relative total TG mass in GMEC after knockdown of $A D R P$ and overexpression of $A T G L$ along with or without OA. Values are means \pm SEM for measurements of 3 wells. Difference in fold change was considered significant when $P<0.05$. Color version available online. 
of the body, its role in lactating ruminant mammary tissue is poorly understood. The ability of $A D R P$ to enhance the synthesis of CLD in GMEC suggests that it plays an important role in regulating CLD formation in mammary cells.

Using proteomics, the overall protein composition of CLD isolated from milk-secreting cells of mice (Wu et al., 2000) and the milk fat layer of cows (Lu et al., 2014) confirmed that ADRP is one of the most abundant proteins on the milk CLD. The fact that ADRP colocalized with CLD in GMEC agrees with previous cell culture experiments (Imamura et al., 2002; McManaman et al., 2007; Krahmer et al., 2009).

In nonruminant hepatocytes, long-chain FA (e.g., OA) stimulate lipid accumulation in parallel with the expression of ADRP (Fan et al., 2009). Data from the present study confirmed this effect in GMEC. The greater colocalization of ADRP with CLD in response to exogenous $\mathrm{OA}$ is suggestive of a role for this protein in binding and transporting intracellular NEFA, hence leading to greater TG and CLD synthesis (Gao and Serrero, 1999).

In ruminants, the mRNA expression of $A D R P$ in mammary tissue closely followed the pattern of milk synthesis and increased remarkably through $\sim 100$ d postpartum (Bionaz and Loor, 2008). The marked increase in the lipid accumulation detected with both Oil Red O staining and immunocytochemical staining confirms previous suggestions that overexpression of $A D R P$ could stimulate CLD formation in fibroblasts (Imamura et al., 2002; Larigauderie et al., 2004). The fact that incubation of cells overexpressing $A D R P$ with OA increased lipid accumulation suggests that ADRP facilitates transport of NEFA within GMEC, which agrees with a previous hypothesis in Cos-7 cell line (Gao and Serrero, 1999; Ohsaki et al., 2008). Mechanistically, the binding of NEFA by ADRP likely contributes to the rapid appearance of FA at the surface of CLD. Whether such a response also is associated with enhanced TG synthesis and subsequent CLD formation (Serrero et al., 2000) is yet to be established.

Knocking out $A D R P$ in fibroblasts did not cause a significant change in lipid accumulation due to the compensatory upregulation of TIP47 protein (Sztalryd et al., 2006), which plays a pivotal role in CLD formation and biogenesis (Bickel et al., 2009; Nose et al., 2013; Patel et al., 2014). The decrease of TG concentration after downregulation of $A D R P$ in our study is consistent with the fact that knocking down both ADRP and TIP 47 decreased CLD formation and increased the ability of TG lipolysis in fibroblasts (Sztalryd et al., 2006). The fact that knockdown of $A D R P$ could not completely eliminate lipid accumulation at a morphological level in the present study is highly suggestive that some unidentified factor (e.g., TIP47, PLIN1, or PLIN4) compensates for ADRP activity in lipid formation. However, the lower TG concentration after knockdown of ADRP indicated that compensatory factors could not completely rescue the effect of ADRP knockdown. Because TIP 47 and PLIN1 have a low expression in goat mammary gland (data not shown), it is speculated that $A D R P$ is most important in CLD formation in goat mammary gland. Further work should be performed to address whether and how other PAT family members work in concert with ADRP to control CLD formation in goat mammary cells.

The present study did not address the exact mechanism whereby ADRP gene and protein promotes the increase of CLD clustering and fusion. The ADRP protein may serve as a factor maintaining the stability of CLD as, for example, PLIN1 in adipocytes (Brasaemle et al., 2000). Previous evidence that ADRP deficiency did not prevent CLD formation but disrupted the increase in CLD size in differentiating mouse mammary epithelial cells (Russell et al., 2011) was highly suggestive that ADRP is a unique factor altering the diameter of CLD. Recently, several protein factors including the cell death-inducing DFF45-like effector family (Yang et al., 2012; Wilfling et al., 2014) and atlastin GTPases (Klemm et al., 2013) were identified as promoting the enlargement of CLD. More experiments are needed to explore the interaction among these proteins in the process of CLD formation and enlargement in ruminant mammary gland.

Adipose triglyceride lipase catalyzes the initial step in the hydrolysis of TG in adipose and other tissues (Zechner et al., 2009). Absence of ATGL in mice increases adipose tissue mass and promotes lipid storage in multiple tissues (Haemmerle et al., 2006). In adipocytes, PLIN1 helps stabilize CLD by forming a barrier that reduces the access of ATGL to TG within CLD, thus inhibiting hydrolysis (Brasaemle et al., 2000). Recent data revealed that ATGL hydrolyzes significant amounts of TG in GMEC (Li et al., 2015), which is consistent with the fact that ATGL altered CLD morphology and made it difficult for CLD to accumulate in the present study. The fact that overexpression of $A D R P$ rescued the decrease in TG concentration and CLD accumulation induced by ATGL agrees with a previous hypothesis that ADRP inhibits TG hydrolysis via preventing ATGL from binding to the CLD surface in HEK 293 cells (Listenberger et al., 2007) and mouse mammary tissue (Russell et al., 2011). These results underscore the importance of ADRP gene and protein in regulating size of CLD and suggest that the potential mechanism by which ADRP mediates CLD enlargement is partly due to preventing ATGL-dependent lipolysis in GMEC. 
A limitation of the present study is that because of the use of a two-dimensional cell culture approach; the cells lacked polarity, which is critical for lipid assembly and secretion (Jin and Amberg, 2000). Thus, the absence of cell polarity may partly explain why the lipid droplets were mainly located paranuclearly (Figures $1 \mathrm{~A}$ and $2 \mathrm{~A}$ ). Despite this limitation, the data still support a role for ADRP gene and protein in ruminant mammary gland. Further in vivo experiments utilizing, for example, mammary tissue at different stages of lactation will have to be conducted to validate the importance of ADRP in the mammary gland.

In summary, our data directly demonstrated that $A D R P$ is a regulator of TG accumulation in the GMEC. The localization of ADRP on CLD surface underscores the important role of ADRP in CLD metabolism and CLD secretion in GMEC. Overexpression of $A D R P$ increases the lipid accumulation, whereas knockdown of $A D R P$ prevented the lipid accumulation. Furthermore, the fact that ADRP rescued the CLD hydrolysis induced by $A T G L$ is highly suggestive of ADRP playing a role in stabilizing CLD in GMEC.

\section{ACKNOWLEDGMENTS}

This work was jointly supported by National Natural Science Foundation of China (31372281), the Transgenic New Species Breeding Program of China (2014ZX08009-051B), and the "Special Fund for Agroscientific Research in the Public Interest (201103038)."

\section{REFERENCES}

Bickel, P. E., J. T. Tansey, and M. A. Welte. 2009. PAT proteins, an ancient family of lipid droplet proteins that regulate cellular lipid stores. Biochim. Biophys. Acta 1791:419-440.

Bionaz, M., and J. Loor. 2008. Gene networks driving bovine milk fat synthesis during the lactation cycle. BMC Genomics 9:366.

Brasaemle, D. L., T. Barber, N. E. Wolins, G. Serrero, E. J. Blanchette-Mackie, and C. Londos. 1997. Adipose differentiation-related protein is an ubiquitously expressed lipid storage droplet-associated protein. J. Lipid Res. 38:2249-2263.

Brasaemle, D. L., G. Dolios, L. Shapiro, and R. Wang. 2004. Proteomic analysis of proteins associated with lipid droplets of basal and lipolytically stimulated 3T3-L1 adipocytes. J. Biol. Chem. 279:46835-46842.

Brasaemle, D. L., B. Rubin, I. A. Harten, J. Gruia-Gray, A. R. Kimmel, and C. Londos. 2000. Perilipin A increases triacylglycerol storage by decreasing the rate of triacylglycerol hydrolysis. J. Biol. Chem. 275:38486-38493.

Chang, B. H.-J., L. Li, A. Paul, S. Taniguchi, V. Nannegari, W. C. Heird, and L. Chan. 2006. Protection against fatty liver but normal adipogenesis in mice lacking adipose differentiation-related protein. Mol. Cell. Biol. 26:1063-1076.

Chong, B. M., P. Reigan, K. D. Mayle-Combs, D. J. Orlicky, and J. L. McManaman. 2011. Determinants of adipophilin function in milk lipid formation and secretion. Trends Endocrinol. Metab. 22:211-217.

Fan, B., S. Ikuyama, J. Q. Gu, P. Wei, J. Oyama, T. Inoguchi, and J. Nishimura. 2009. Oleic acid-induced ADRP expression requires both AP-1 and PPAR response elements, and is reduced by Pycnogenol through mRNA degradation in NMuLi liver cells. Am. J. Physiol. Endocrinol. Metab. 297:E112-E123.

Gao, J., and G. Serrero. 1999. Adipose differentiation related protein (ADRP) expressed in transfected COS-7 cells selectively stimulates long chain fatty acid uptake. J. Biol. Chem. 274:16825-16830.

Haemmerle, G., A. Lass, R. Zimmermann, G. Gorkiewicz, C. Meyer, J. Rozman, G. Heldmaier, R. Maier, C. Theussl, S. Eder, D. Kratky, E. F. Wagner, M. Klingenspor, G. Hoefler, and R. Zechner. 2006. Defective lipolysis and altered energy metabolism in mice lacking adipose triglyceride lipase. Science 312:734-737.

Hansen, H. O., I. Grunnet, and J. Knudsen. 1984. Triacylglycerol synthesis in goat mammary gland. The effect of ATP, Mg2+ and glycerol 3-phosphate on the esterification of fatty acids synthesized de novo. Biochem. J. 220:513-519.

Imamura, M., T. Inoguchi, S. Ikuyama, S. Taniguchi, K. Kobayashi, N. Nakashima, and H. Nawata. 2002. ADRP stimulates lipid accumulation and lipid droplet formation in murine fibroblasts. Am. J. Physiol. Endocrinol. Metab. 283:E775-E783.

Jiang, H. P., and G. Serrero. 1992. Isolation and characterization of a full-length cDNA coding for an adipose differentiation-related protein. Proc. Natl. Acad. Sci. USA 89:7856-7860.

Jin, H., and D. C. Amberg. 2000. The secretory pathway mediates localization of the cell polarity regulator Aip3p/Bud6p. Mol. Biol. Cell 11:647-661.

Kang, Y., S. Hengbo, L. Jun, L. Jun, Z. Wangsheng, T. Huibin, and S. Huaiping. 2015. PPARG modulated lipid accumulation in dairy GMEC via regulation of ADRP gene. J. Cell. Biochem. 116:192201

Klemm, R. W., J. P. Norton, R. A. Cole, C. S. Li, S. H. Park, M. M. Crane, L. Li, D. Jin, A. Boye-Doe, T. Y. Liu, Y. Shibata, H. Lu, T. A. Rapoport, R. V. Farese Jr., C. Blackstone, Y. Guo, and H. Y. Mak. 2013. A conserved role for atlastin GTPases in regulating lipid droplet size. Cell Reports 3:1465-1475.

Krahmer, N., Y. Guo, R. V. Farese Jr., and T. C. Walther. 2009. SnapShot: lipid droplets. Cell 139:1024-1024.e1.

Larigauderie, G., C. Cuaz-Pérolin, A. B. Younes, C. Furman, C. Lasselin, C. Copin, M. Jaye, J.-C. Fruchart, and M. Rouis. 2006. Adipophilin increases triglyceride storage in human macrophages by stimulation of biosynthesis and inhibition of $\beta$-oxidation. FEBS J. 273:3498-3510.

Larigauderie, G., C. Furman, M. Jaye, C. Lasselin, C. Copin, J.-C. Fruchart, G. Castro, and M. Rouis. 2004. Adipophilin enhances lipid accumulation and prevents lipid efflux from THP-1 macrophages: Potential role in atherogenesis. Arterioscler. Thromb. Vasc. Biol. 24:504-510

Li, J., J. Luo, H. Wang, H. Shi, J. Zhu, Y. Sun, K. Yu, and D. Yao. 2015. Adipose triglyceride lipase regulates lipid metabolism in dairy goat mammary epithelial cells. Gene 554:125-130.

Lin, X. Z., J. Luo, L. Zhang, W. Wang, H. Shi, and J. Zhu. 2013. MiR27a suppresses triglyceride accumulation and affects gene mRNA expression associated with fat metabolism in dairy goat mammary gland epithelial cells. Gene 521:15-23.

Listenberger, L. L., A. G. Ostermeyer-Fay, E. B. Goldberg, W. J. Brown, and D. A. Brown. 2007. Adipocyte differentiation-related protein reduces the lipid droplet association of adipose triglyceride lipase and slows triacylglycerol turnover. J. Lipid Res. 48:27512761.

Lu, J., T. van Hooijdonk, S. Boeren, J. Vervoort, and K. Hettinga. 2014. Identification of lipid synthesis and secretion proteins in bovine milk. J. Dairy Res. 81:65-72.

Maller, O., H. Martinson, and P. Schedin. 2010. Extracellular matrix composition reveals complex and dynamic stromal-epithelial interactions in the mammary gland. J. Mammary Gland Biol. Neoplasia 15:301-318.

McManaman, J. L. 2012. Milk lipid secretion: Recent biomolecular aspects. Biomol. Concepts 3:581-591.

McManaman, J. L., T. Russell, J. Schaack, D. Orlicky, and H. Robenek. 2007. Molecular determinants of milk lipid secretion. J. Mammary Gland Biol. Neoplasia 12:259-268. 
Mehlem, A., C. E. Hagberg, L. Muhl, U. Eriksson, and A. Falkevall. 2013. Imaging of neutral lipids by oil red O for analyzing the metabolic status in health and disease. Nat. Protoc. 8:1149-1154.

Motomura, W., M. Inoue, T. Ohtake, N. Takahashi, M. Nagamine, S. Tanno, Y. Kohgo, and T. Okumura. 2006. Up-regulation of ADRP in fatty liver in human and liver steatosis in mice fed with high fat diet. Biochem. Biophys. Res. Commun. 340:1111-1118.

Nose, F., T. Yamaguchi, R. Kato, T. Aiuchi, T. Obama, S. Hara, M. Yamamoto, and H. Itabe. 2013. Crucial role of perilipin-3 (TIP47) in formation of lipid droplets and PGE2 production in HL-60-derived neutrophils. PLoS ONE 8:e71542.

Ohsaki, Y., J. Cheng, M. Suzuki, A. Fujita, and T. Fujimoto. 2008. Lipid droplets are arrested in the ER membrane by tight binding of lipidated apolipoprotein B-100. J. Cell Sci. 121:2415-2422.

Oppi-Williams, C., J. K. Suagee, and B. A. Corl. 2013. Regulation of lipid synthesis by liver $\mathrm{X}$ receptor alpha and sterol regulatory element-binding protein 1 in mammary epithelial cells. J. Dairy Sci. 96:112-121.

Patel, S., W. Yang, K. Kozusko, V. Saudek, and D. B. Savage. 2014. Perilipins 2 and 3 lack a carboxy-terminal domain present in perilipin 1 involved in sequestering ABHD5 and suppressing basal lipolysis. Proc. Natl. Acad. Sci. USA 111:9163-9168.

Reinhardt, T. A., and J. D. Lippolis. 2006. Bovine milk fat globule membrane proteome. J. Dairy Res. 73:406-416.

Russell, T. D., C. A. Palmer, D. J. Orlicky, A. Fischer, M. C. Rudolph, M. C. Neville, and J. L. McManaman. 2007. Cytoplasmic lipid droplet accumulation in developing mammary epithelial cells: Roles of adipophilin and lipid metabolism. J. Lipid Res. 48:14631475 .

Russell, T. D., J. Schaack, D. J. Orlicky, C. Palmer, B. H.-J. Chang, L. Chan, and J. L. McManaman. 2011. Adipophilin regulates maturation of cytoplasmic lipid droplets and alveolae in differentiating mammary glands. J. Cell Sci. 124:3247-3253.

Serrero, G., A. Frolov, F. Schroeder, K. Tanaka, and L. Gelhaar. 2000. Adipose differentiation related protein: expression, purification of recombinant protein in Escherichia coli and characterization of its fatty acid binding properties. Biochim. Biophys. Acta 1488:245254.

Shi, H., J. Zhu, J. Luo, W. Cao, H. Shi, D. Yao, J. Li, Y. Sun, H. Xu, $\mathrm{K}$. Yu, and J. Loor. 2015. Genes regulating lipid and protein me- tabolism are highly expressed in mammary gland of lactating dairy goats. Funct. Integr. Genomics 15:309-321.

Shi, H. B., J. Luo, D. W. Yao, J. J. Zhu, H. F. Xu, H. P. Shi, and J. J. Loor. 2013a. Peroxisome proliferator-activated receptor-Y stimulates the synthesis of monounsaturated fatty acids in dairy goat mammary epithelial cells via the control of stearoyl-coenzyme A desaturase. J. Dairy Sci. 96:7844-7853.

Shi, H., J. Luo, J. J. Zhu, J. Li, Y. T. Sun, X. Z. Lin, L. P. Zhang, D. W. Yao, and H. P. Shi. 2013b. PPAR $\gamma$ regulates genes involved in triacylglycerol synthesis and secretion in mammary gland epithelial cells of dairy goats. PPAR Res. 2013:310948.

Shi, H. B., W. S. Zhao, J. Luo, D. W. Yao, Y. T. Sun, J. Li, H. P. Shi and J. J. Loor. 2014. Peroxisome proliferator-activated receptor $\gamma 1$ and $\gamma 2$ isoforms alter lipogenic gene networks in goat mammary epithelial cells to different extents. J. Dairy Sci. 97:5437-5447.

Sztalryd, C., M. Bell, X. Lu, P. Mertz, S. Hickenbottom, B. H.-J Chang, L. Chan, A. R. Kimmel, and C. Londos. 2006. Functional compensation for adipose differentiation-related protein (ADFP) by Tip47 in an ADFP null embryonic cell line. J. Biol. Chem. 281:34341-34348.

Thiele, C., and J. Spandl. 2008. Cell biology of lipid droplets. Curr. Opin. Cell Biol. 20:378-385.

Wang, Z., J. Luo, W. Wang, W. Zhao, and X. Lin. 2010. Characterization and culture of isolated primary dairy goat mammary gland epithelial cells. Sheng Wu Gong Cheng Xue Bao 26:1123-1127.

Wilfling, F., J. T. Haas, T. C. Walther, and R. V. F. Jr. 2014. Lipid droplet biogenesis. Curr. Opin. Cell Biol. 29:39-45.

Wu, C. C., K. E. Howell, M. C. Neville, J. R. Yates, and J. L. McManaman. 2000. Proteomics reveal a link between the endoplasmic reticulum and lipid secretory mechanisms in mammary epithelial cells. Electrophoresis 21:3470-3482.

Yang, H., A. Galea, V. Sytnyk, and M. Crossley. 2012. Controlling the size of lipid droplets: lipid and protein factors. Curr. Opin. Cell Biol. 24:509-516.

Zechner, R., P. C. Kienesberger, G. Haemmerle, R. Zimmermann, and A. Lass. 2009. Adipose triglyceride lipase and the lipolytic catabolism of cellular fat stores. J. Lipid Res. 50:3-21. 